\title{
Study of Lipid Profile in Ischemic Cerebrovascular Disease
}

\author{
Authors \\ Dr G. Pragathi ${ }^{1}$, Dr Dileep M Rampure ${ }^{2}$, Dr G.Sreeram ${ }^{3}$ \\ ${ }^{1,3}$ Post Graduate, ${ }^{2} \mathrm{MD}$, Prof and Head of Dept \\ Corresponding Author \\ Dr G. Pragathi \\ Post Graduate \\ Email:dr.g.pragathi@gmail.com
}

\begin{abstract}
:
Introduction: Stroke is defined as abrupt onset of a neurological deficit that is attributable to a focal vascular cause. The role of circulating lipids and lipoproteins in the pathogenesis of ischemic stroke remains uncertain despite three decades of research.

Aim:1) To evaluate lipid profile 2) To study the association between serum lipids and ischemic stroke Materials and Methods: This study was carried out in Mamata General \& Super Specialty Hospital, attached to Mamata Medical College, between September 2013 and August 2015 in 50 patients of >35 years of age.
\end{abstract}

Results: The youngest patient in the study was 35 years old and the eldest was 83 years old. Maximum number of patients were in age group $\geq 65.60 \%$ of the cases were males and $40 \%$ of patients were females. There is male preponderance.MCA is involved in 58\% cases. Out of 30 males 4, and out of 20 females 7 , patients had hyperlipidemia. Hyperlipidemia was 3rd major risk factor (22\%) for stroke after hypertension and smoking.

Conclusion: Thus prevention of atherosclerosis rather than treatment is the goal. Drug treatment for hyperlipidemia should be individualized based on the calculated risk and as per latest available guidelines.

KEY WORDS: Ischemic cerebro vascular disease (ICD), Stroke, Hyperlipidemia.

\section{INTRODUCTION}

Stroke is defined as 'abrupt onset of a neurological deficit that is attributable to a focal vascular cause ${ }^{1}$. The WHO clinically defines stroke as 'the rapid development of clinical signs and symptoms of a focal neurological disturbance lasting more than 24 hours or leading to death with no apparent cause other than vascular origin'.
It is the second commonest cause of death and fourth leading cause of disability worldwide ${ }^{2}$. The role of circulating lipids and lipoproteins in the pathogenesis of ischemic stroke remains uncertain despite three decades of research. The relation of circulating cholesterol to ischemic stroke does not resemble its well-known relation to coronary heart disease. Either the pathogenesis of a substantial 
proportion of ischemic strokes does not involve classic atherosclerotic mechanisms, or the effect of plasma lipids on atherogenesis is substantially different in the intracranial vascular bed ${ }^{3}$. Epidemiological studies have shown that patients with elevated total cholesterol, LDL cholesterol and decreased HDL cholesterol are at higher risk of developing atherothrombotic stroke. The current study was done to evaluate the association of serum lipid profile with ischemic stroke. ${ }^{2}$

\section{MATERIALS AND METHODS}

This study was carried out in Mamata General \& Super Specialty Hospital, attached to Mamata Medical College, between September 2012 and August 2014. A total of 50 patients aged more than 35 years were included. The youngest patient in the study was 35 years old and the eldest was 83 years old. Maximum number of patients were in group $\geq 65.30$ male and 20 females diagnosed to have ischemic cerebrovascular disease have been the subjects of the study. Age and sex matched 50 healthy persons were taken as controls.

\section{INCLUSION CRITERIA}

1. Abrupt or relatively rapid onset of focal neurological disturbance which persisted for more than 24 hours.

2. Gradual onset with smooth or stuttering progression over few hours.

3. CT scan brain evidence of infarction.

\section{EXCLUSION CRITERIA}

1. Cerebral infarction associated with head injury, pregnancy, puerperium, dehydration, infections, hematological disturbances, uraemic and hepatic encephalopathy, with previous neurological deficits and TIAs.

2. CT scan evidence of old lacunar infarcts, intracerebral hemorrhage, patients who were on lipid lowering drugs and who came after the first week of onset of stroke were excluded.
The purpose of elimination was to obtain a pure picture of the relationship between ischemic cerebrovascular disease and serum lipids. Samples for analysis were taken in the first week of post cerebral infarction period.

Each patient was subjected to thorough clinical examination, CT scan brain, Hb, TC, DC, ESR, fasting blood sugar, blood urea, Serum.Creatinine, Fasting Lipid profile was done in all cases.

Blood was sampled for Biochemical assay of

- Serum total cholesterol

- Serum triglycerides

- High density lipoprotein

- Low density lipoprotein

- Very low density lipoprotein

\section{RESULTS}

The present study with 50 patients has the youngest who was 35 years old and the eldest was 83 years. Maximum number of patients were in group $\geq 65$ with Mean age of $59.7 \pm 13.02$ yrs.60\% of the cases were males and $40 \%$ of patients were females. There is male preponderance. MCA was involved in 58\%, PCA was involved in $32 \%$ and ACA was involved in $10 \%$ of cases.

\section{LIPID PROFILE IN STUDY AND CONTROL GROUPS}

\begin{tabular}{|c|c|c|c|c|c|c|c|}
\hline & & TC & TG & VLDLc & LDLc & HDLc & HDLc/TC \\
\hline \multirow{2}{*}{ Study } & Mean & 208.551 & 171.8 & 35 & 139.88 & 34.4 & 0.172 \\
& $\mathrm{SD}$ & \pm & \pm & \pm & \pm & \pm & \pm \\
& Range & 42.526 & 49.468 & 10.996 & 38.125 & 4.544 & 0.042 \\
\hline \multirow{2}{*}{ Control } & Mean & 157.78 & 132.92 & 26.792 & 87.468 & 43.14 & 0.281 \\
& RD & \pm & \pm & \pm & \pm & \pm & \pm \\
& Range & 20.553 & 12.901 & 2.954 & 21.505 & 3.307 & 0.042 \\
\hline \multirow{2}{*}{ Study } & T - & 7.284 & 5.377 & 8.398 & 8.466 & 10.995 & 12.701 \\
Vs & & & & & & \\
\cline { 2 - 8 } Control & $\mathrm{P}-$ & $<0.01$ & $<0.01$ & $<0.01$ & $<0.01$ & $<0.01$ & $<0.01$ \\
& value & $(\mathrm{S})$ & $(\mathrm{S})$ & $(\mathrm{S})$ & $(\mathrm{S})$ & $(\mathrm{S})$ & $(\mathrm{S})$ \\
\hline
\end{tabular}

This shows comparison of mean and standard deviation range of Total Cholesterol (TC), triglycerides (TG), VLDLc, LDLc, HDLc and HDLc/TC. Calculated T- value and P- value $(<0.01)$ which was significant. Further, Out of 30 
male 4 and out of 20 female patients 7 patients had hyperlipidemia.

\section{TYPES OF HYPERLIPIDEMIA}

\begin{tabular}{|c|c|c|c|c|}
\hline $\begin{array}{c}\text { Type of } \\
\text { hyperlipidemia }\end{array}$ & Male & Female & Total & Percentage \\
\hline Ilb & 04 & 05 & 09 & 81.8 \\
\hline Ila & - & 01 & 01 & 9.09 \\
\hline IV & - & 01 & 01 & 9.09 \\
\hline Total & 04 & 07 & 11 & 100 \\
\hline
\end{tabular}

This shows incidence of types of hyperlipidemia. A total of 11 patients have hyperlipidemia. Out of them 4 male and 5 female patients (total 9 patients, $81.8 \%$ ) have Type IIbhyperlipidemia and one female patient $(9.09 \%)$ has type IIa and one female patient $(9.09 \%)$ has type IV hyperlipidemia.

\section{DISCUSSION}

Stroke is the third leading cause of death after heart diseases and cancer in the developed countries with an average incidence of approximately 1 in 1000 population. Patients with elevated lipid levels are at higher risk for atherothrombotic brain infarction than those with normal values and this applies mainly to premature ischemic CVD. So hyperlipidemia has emerged clearly as a major risk factor associated with increased risk of atherosclerosis either alone or in combination with other risk factors like smoking and hypertension.

\section{Mean age of incidence:}

In the present study the peak incidence of ICD was $>65$ years contributing $40 \%$. Mean age with standard deviation was $59.7 \pm 13$.

\section{Sex distribution:}

In the present study male: Female ratio was 2.1:1. This was comparable with other studies in India. Men are more likely to have a stroke than women: the male/female sex ratio for India is 7:1.This may be due to differences in risk factors such as smoking and drinking ${ }^{4}$. The mean onset of stroke for men in India ranges from 63-65 and 57- 68years for women ${ }^{5}$.Results showed that female stroke patients were older than male stroke patients and were more likely to have transient ischemic attack and hypertension.

Comparison of lipid levels between ischemic cerebrovascular disease patients and controlsTotal Cholesterol:

The value of total cholesterol was significantly elevated in patients with ICD when compared to controls in present study. $(\mathrm{P}<0.001)$. This observation supports the earlier reports.

In other study i.e. Alok Mohankar.et.al ${ }^{6}$ in 1993 showed elevated levels of total cholesterol predispose to large vessel atherothrombotic and in other study i.e. Linden Strorn $E^{7}$ in 1994 reported that decreased total cholesterol reduces the incidence of stroke in coronary heart disease patients.

The MR FIT (Multiple Risk Factor Intervention Trial) showed that the risk for ischemic stroke increased as TC levels exceeded $200 \mathrm{mg} / \mathrm{dl}$. In fact, the risk for ischemic stroke more than doubled when TC levels exceeded $280 \mathrm{mg} / \mathrm{dl}^{8,9}$.

\section{Triglycerides}

The serum triglyceride levels were significantly higher in cases of ICD than in controls. This observation is in full agreement with other studies. High TG: HDL-C ratio was associated with symptomatic intracranial atherosclerotic disease and may represent a therapeutic target for optimizing intensive medical treatment ${ }^{10}$.

HDLc:

Mounting clinical and experimental evidence shows that HDLs exert multiple anti-atherogenic and antithrombotic effects that together are consistent with a marked reduction in the risk of a morbid cardiovascular event. These findings support the use of therapeutic strategies to counter the common finding of low HDL-cholesterol in patients with dyslipidemia ${ }^{11}$.

The levels of serum HDLc, which was significantly decreased in cases of ICD when compared to control $(\mathrm{P}<0.001)$ are consistent with a large volume of publications on this subject. 


\section{LDLc:}

LDL is believed to be the most atherogenic lipoprotein, majority(upto 50\%) of cholesterol in plasma is found in LDL. Statin therapy with intensive lipid-lowering effects is recommended to reduce risk of stroke and cardiovascular events among patients who have evidence of atherosclerosis. For patients with atherosclerotic ischemic stroke or TIA and without known CHD, it is reasonable to target a reduction of at least $50 \%$ in LDL-C or a target LDL-C level of 70 $\mathrm{mg} / \mathrm{dl}$ to obtain maximum benefit ${ }^{12}$.

The present study revealed that significant increase in levels of serum LDLc in ICD patients, when compared to controls $(\mathrm{P}<0.001)$. This is in accordance with Kurth T.et.al study ${ }^{13}$ in 2007 . In view of the present study results, total cholesterol was a risk factor in ischemic stroke whereas high TG levels had a protective role against hemorrhagic events. As a result, treating high LDL levels can be a helpful option to reduce these events and eventually decrease the related morbidity and mortality rates.

\section{VLDLc:}

Liver secretes very low-density lipoproteins (VLDLs) that are rich in triglyceride and relatively poor in cholesterol. The endothelial enzyme, lipoprotein lipase, converts much of the triglycerides in VLDLs to free fatty acids, which are used by peripheral tissues as an energy source. VLDLc levels were significantly elevated in ICD patients when compared to controls in present study $(\mathrm{P}<0.001)$ which is an established risk factor for ICD.

\section{Incidence and type of hyperlipidemia:}

Type IIbhyperlipidemia is observed in 9 patients, 5 of them are females and 4 are males. Type IIa in 1 female patient and type IV in one female patient.

\section{CONCLUSION}

- Thus prevention of atherosclerosis rather than treatment is the goal.

- Drug treatment for hyperlipidemia should be individualized based on the calculated risk and as per latest available guidelines.

\section{REFERENCES}

1. Harrison's principles of internal medicine, eighteenth edition, page 3270.

2. Strong K, Mathers C, Bonita R. Preventing stroke: saves lives around the world. Lancet Neurol 2007; 6:182-7

3. Eyal Shahar, MD, MPH; Lloyd E. Chambless, PhD; Wayne D. Rosamond, $\mathrm{PhD}$; Lori L. Boland, MPH; Christie M. Ballantyne, MD; Paul G. McGovern, PhD†; A. Richey Sharrett, MD, DrPH . Plasma Lipid Profile and Incident Ischemic StrokeThe Atherosclerosis Risk in Communities (ARIC) Study. Stroke. 2003; 34:623-631.

4. Das S, Banerjee T. Stroke Indian Scenario. Circulation 2008; 118: 2719-24.

5. Bhattacharya S, Prasarsaha S, Basu A, Das K. A 5 year prospective study of incidence, morbidity and mortality stroke profile on stroke in a rural community of Eastern India. J Indian Med Assoc 2005; 103(12): 655-9.

6. Alok Mohankar, Ravindra Kumar Garg et al. Serum Lipids and Stroke. Neurology India 1993; 41 (1): 1-5.

7. Linden Strorn E, Boysen G, Nyboe J et al. Influence of total cholesterol, high density lipoprotein cholesterol and triglycerides on risk of cerebrovascular study. The Copenhagen City Heart Study. BMJ 1994; 309: 11-15.

8. Iso $\mathrm{H}$, Jacobs DR, Wentworth $\mathrm{D}$, Neaton JD, Cohen JD. Serum cholesterol levels and six-year mortality from stroke in 350,977 men screened for the Multiple Risk Factor Intervention Trial. N Engl J Med. 1989;320(14):904-910.

9. Tirschwell DL, Smith NL, Heckbert SR, Lemaitre RN, Longstreth WT Jr, Psaty BM. Association of cholesterol with stroke risk varies in stroke subtypes and patient subgroups. Neurology. 2004;63(10): 1868-1875. 
10. Vishal B Jani, Saqib S Chaudhry, VikramJadhav, Sayed Hussain, Adnan Safdar, and Adnan I Qureshi, Stroke. 2014; 45:AWP338.

11. Philip Barter, European Heart Journal Supplements (2005) 7 (Supplement F), F4-F8 doi:10.1093/ eurheartj/sui036.

12. Karen L. Furie, Scott E. Kasner, Robert J. Adams, Gregory W. Albers, Ruth L. Bush, Susan C., Stroke. 2010, doi:10.1161/STR.0b013e3181f7d043.

13. Kurth T, Everett BM, Buring JE, Kase CS, Ridker PM, Gaziano JM. Lipid levels and the risk of ischemic stroke in women. Neurology. 2007;68(8):556-562. 